\title{
Bemerkenswerte, aktuelle Publikationen mit österreichischer Beteiligung
}

Clinical indications for image guided interventional procedures in the musculoskeletal system: a Delphi-based consensus paper from the European Society of Musculoskeletal Radiology (ESSR) - part III, nerves of the upper limb. Sconfienza LM, Adriaensen M, Albano D, Allen G, Aparisi Gómez MP, Bazzocchi A, Beggs I, Bignotti B, Chianca V, Corazza A, Dalili D, De Dea M, Del Cura JL, Di Pietto F, Drakonaki E, Facal de Castro F, Filippiadis D, Gielen J, Gitto S, Gupta H, Klauser AS, Lalam R, Martin S, Martinoli C, Mauri G, McCarthy C, McNally E, Melaki K, Messina C, Mirón Mombiela R, Neubauer B, Obradov M, Olchowy C, Orlandi D, Gonzalez RP, Rutkauskas S, Snoj Z, Tagliafico AS, Talaska A, Vasilevska-Nikodinovska V, Vucetic J, Wilson D, Zaottini F, Zappia M, Plagou A; Ultrasound and Interventional Subcommittees of the European Society of Musculoskeletal Radiology (ESSR). Eur Radiol 2020 Mar; 30(3): 1498-1506.

Image-guided interventional procedures of the nerves are commonly performed by physicians from different medical specialties, although there is a lack of clinical indications for these types of procedures. This Delphi-based consensus provided a list of indications on image-guided interventional procedures for nerves of the upper limb based on updated published evidence.

An expert panel of 45 members of the Ultrasound and Interventional Subcommittees of the ESSR participated in this Delphi-based consensus study. After revision of the published papers on image-guided interventional procedures for nerves of the upper limb updated to September 2018 , the experts drafted a list of statements according to the Oxford Centre for evidence-based medicine levels of evidence. Consensus on statements regarding clinical indications was considered as strong when more than $95 \%$ of experts agreed, and broad if more than $80 \%$ agreed.

Ten statements were drafted on procedures for nerves of the upper limb. Only two statements reached the highest level of evidence (ultrasound guidance is a safe and effective method for brachial plexus block; ultrasound-guided non-surgical approaches are safe and effective methods to treat carpal tunnel syndrome in the short term, but there is sparse evidence on the mid- and long-term effectiveness of these interventions). Strong consensus was obtained on $6 / 10$ statements (60\%), while $4 / 10$ statements reached broad consensus (40\%).

This Delphi-based consensus study reported poor evidence on image-guided interventional procedures for nerves of the upper limb. Sixty percent of statements on clinical indications provided by the expert board reached a strong consensus.

Cutaneous nerve fields of the anteromedial lower limb-Determination with selective ultrasound-guided nerve blockade. Riegler G, Pivec C, Jengojan S, Mayer JA, Schellen C, Trattnig S, Bodner G. Clin Anat. 2020 Feb 17. doi:10.1002/ca.23 582.

This study aimed to determine the peripheral cutaneous nerve fields (CNF), their variability, and potential overlap by selectively blocking the intermediate (IFCN) and medial (MFCN) femoral cutaneous nerves and the infrapatellar branch of the saphenous nerve (IPBSN) in healthy volunteers.

In this prospective study, ultrasound-guided nerve blockades of the IFCN, MFCN, and IPBSN in 14 healthy volunteers were administered. High-frequency probes (15$22 \mathrm{MHz}$ ) and $1 \mathrm{ml}$ of $1 \%$ lidocaine per nerve were used. The area of sensory loss was determined using a pinprick, and all fields were drawn on volunteers' skin. A threedimensional (3D) scan of all lower limbs was obtained and the three CNF and their potential overlap were measured.

The mean size of innervation areas showed a high variability of peripheral CNF, with $258.58 \pm 148.26 \mathrm{~mm}^{2}$ (95\% Cl, 169$348.18 \mathrm{~mm}^{2}$ ) for the IFCN, $193.26 \pm$ $72.08 \mathrm{~mm}^{2}\left(95 \% \mathrm{Cl}, 124.45-262.08 \mathrm{~mm}^{2}\right)$ for the MFCN, and $166.78 \pm 121.30 \mathrm{~mm}^{2}$ ( $95 \% \mathrm{Cl}, 94.1-239.46 \mathrm{~mm}^{2}$ ) for the IPBSN. In 11 volunteers, we could evaluate an overlap between the IFCN and MFCN (range, $4.11-139.68 \pm 42.70 \mathrm{~mm}^{2}$ ), and, in 10 volunteers, between the MFCN and IPBSN (range, 11.12-224.95 $\pm 79.61 \mathrm{~mm}^{2}$ ). In only three volunteers was an overlap area found between the IFCN and IPBSN (range, $7.46-224.95 \pm 88.88 \mathrm{~mm}^{2}$ ). The 3D-scans confirmed the high variability of the peripheral CNF.

Our study successfully determined CNF, their variability, and the overlap of the MFCN, IFCN, and IPBSN in healthy volunteers. Therefore, we encourage physicians to use selective nerve blockades to correctly determine peripheral CNF at the anteromedial lower limb.

The Fetal Posterior Fossa on Prenatal Ultrasound Imaging: Normal Longitudinal Development and Posterior Fossa Anomalies. Pertl $B^{1}$, Eder $S^{1}$, Stern $C^{1}$, Verheyen $S^{2}$. Ultraschall in Med 2019 Dec; 40(6): 692721.

Fetal neurosonography and the assessment of the posterior fossa have gained in importance during the last 2 decades primarily due to the development of high-resolution ultrasound probes and the introduction of 3D sonography. The anatomical development of the posterior fossa can be visualiz- 
ed well with the newest ultrasound technologies. This allows better knowledge of the anatomical structures and helps with understanding of the development of malformations of the posterior fossa. In this article the longitudinal development of the posterior fossa structures will be reviewed. The embryologic description will be compared with ultrasound descriptions. These embryologic and anatomic illustrations form the basis for the screening and diagnosis of malformations of the posterior fossa. During the first trimester, screening for open spina bifida as well as cystic malformations of the posterior fossa is possible. In the second and third trimester, malformations of the posterior fossa can be subdivided into 3 groups: fluid accumulation in the posterior fossa (Dandy-Walker malformation, Blake's pouch cyst, mega cisterna magna, arachnoid cyst, vermian hypoplasia), decreased cerebellar biometrics (volume) (cerebellar hypoplasia, pontocerebellar hypoplasia) and suspicious cerebellar anatomy (Arnold-Chiari malformation, rhombencephalosynapsis, Joubert syndrome). This algorithm, in combination with knowledge of normal development, facilitates the diagnostic workup of malformations of the posterior fossa.

EFSUMB Gastrointestinal Ultrasound (GIUS) Task Force Group: Celiac sprue and other rare gastrointestinal diseases ultrasound features. Dietrich CF, Hollerweger A, Dirks K, Higginson A, Serra C, Calabrese E, Dong Y, Hausken T, Maconi G, Mihmanli I, Nürnberg D, Nylund K, Pallotta N, Ripollés T, Romanini L, Săftoiu A, Sporea I, Wüstner M, Maaser C, Gilja OH. Med Ultrason 2019 Aug 31; 21(3): 299-315.

Transabdominal gastrointestinal ultrasound (GIUS) is unique in its capacity to examine the bowel non-invasively and in its physiological condition, including extra-intestinal features such as the splanchnic vessels, mesentery, omentum and lymph nodes- even at the bedside. Despite this, and its extensive documentation for its usefulness, it has only been fully implemented in a few European countries and expert centres. Therefore, the European Federation of Societies for Ultrasound in Medicine and Biology (EFSUMB) established a GIUS Task
Force Group in 2014 consisting of international experts from 9 European countries with the objectives to standardize and promote the use of GIUS in a clinical setting. This is achieved by publishing clinical guidelines and recommendations on indications and use of GIUS and so far, 4 guidelines have been published: first on "examination techniques and normal findings", second on "inflammatory bowel disease", third on "acute appendicitis and diverticulitis" and fourth on "transrectal and perineal ultrasound". This paper describes the ultrasound features of miscellaneous disorders such as celiac disease, cystic fibrosis, omental infarction, Meckel's diverticle, endometriosis, intestinal neoplasia, mucocele, amyloidosis, GVHD, foreign bodies, vasculitis, and pneumatosis cystoides intestinalis. Bowel ultrasound can be indicated in most of these conditions to investigate intestinal symptoms but in other cases the alterations of the bowel can be also an incidental finding that suggest other examinations which finally help to discover an unknown pathological condition.

EFSUMB Position Paper: Recommendations for Gastrointestinal Ultrasound (GIUS) in Acute Appendicitis and Diverticulitis. Dirks $\mathrm{K}$, Calabrese E, Dietrich CF, Gilja OH, Hausken T, Higginson A, Hollerweger A, Maconi G, Maaser C, Nuernberg D, Nylund K, Pallotta N, Ripolles T, Romanini L, Saftoiu A, Serra C, Wüstner M, Sporea I. Ultraschall in Med 2019 Apr; 40(2): 163-175.

An interdisciplinary task force of European experts summarizes the value of gastrointestinal ultrasound (GIUS) in the management of acute appendicitis and diverticulitis. Based on an extensive literature review, clinical recommendations for these highly common diseases in visceral medicine are presented.In patients with acute appendicitis, preoperative sonography has been established as a routine procedure in most European countries for medical and legal reasons. Routine sonography in these patients may reduce the rate of unnecessary surgery by half. The sensitivity, specificity, and accuracy of ultrasound reach values above $90 \%$ and are equivalent to CT and MRI. However, the high operator dependence may be a problem, for example in point-of-care ultrasound in emergency departments. Structured training programs, quality controls and standardized ultrasound reporting should be increasingly implemented.In the case of suspected acute diverticulitis, "ultrasound first" should also be a basic element in the approach to all patients. Sonography can confirm the diagnosis and allows early risk stratification. As treatment strategies have become less aggressive and more tailored to the stage of diverticulitis, accurate staging has become increasingly important. GIUS and CT have proven to have similar sensitivity and specificity. Especially in cases of uncomplicated diverticulitis, GIUS will be the one and only imaging procedure. CT may work as a backup and has particular advantages for diverticulitis located in the distal sigmoid, inflammation deep in the small pelvis and insufficient ultrasound scanning conditions. This step-up approach (ultrasound first and CT only in case of a negative or inconclusive ultrasound result) has proven to yield the best accuracy.

The Use of Handheld Ultrasound Devices An EFSUMB Position Paper. Nielsen MB, Cantisani V, Sidhu PS, Badea R, Batko T, Carlsen J, Claudon M, Ewertsen C, Garre C, Genov J, Gilja OH, Havre R, Kosiak M, Kosiak W, Pilcher J, Prosch H, Radzina M, Rafailidis V, Rykkje A, Serra A, Sotiriadis A, Østergaard M, Dietrich. CF Ultraschall in Med. 2019 Feb; 40(1): 30-39.

The miniaturization of ultrasound equipment in the form of tablet- or smartphonesized ultrasound equipment is a result of the rapid evolution of technology and handheld ultrasound devices (HHUSD). This position paper of the European Federation of Societies in Ultrasound and Medicine (EFSUMB) assesses the current status of HHUSD in abdominal ultrasound, pediatric ultrasound, targeted echocardiography and heart ultrasound, and we will report position comments on the most common clinical applications. Also included is a SWOT (Strength - Weaknesses - Opportunities - Threats) analysis, the use for handheld devices for medical students, educational \& training aspects, documentation, storage and safety considerations. 